\title{
SEDIMENTOLOGY OF STREAM SEDIMENTS ALONG THE BED OF FAMGBE RIVER IN YENAGOA AREA OF BAYELSA STATE, NIGERIA
}

\author{
Didei, Innocent Sunday \& Akpofure, Edirin \\ Department of Geology, Faculty of Science, Niger Delta University, Amamssoma Bayelsa State \\ Email: dideiinnocent@gmail.com
}

Keywords: Famgbe River, Grain Size Analysis, Statistical Analysis, Mean Value

\begin{abstract}
Sedimentological studies of samples from Famgbe River were carried out with aid of a mechanical sieve method. 10 loose sediment samples collected along the bank of Famgbe River were subjected to granulometric analysis to determine their textural characteristics and depositional processes. Standard sedimentological methods involving sieve analysis were utilized to determine the various size distribution of each sample. This was further subjected to statistical analysis (mean size, sorting, kurtosis, skeweness). Results show that the samples were very coarse to coarse grain in size $(-0.77 \phi$ to $0.77 \phi)$, poorly to moderately sorted $(0.53 \phi-1.48 \phi)$, nearly symmetrical to positively skewed ($0.01 \phi$ to $0.25 \phi)$ and very platykurtic to leptokurtic $(0.14 \phi$ t0 $1.4 \phi)$ with dominantly coarse grained. Most of the samples are moderately sorted, positively skewed and leptokurtic nature of sediments. This suggests that the sediments were transported by saltation and traction and being deposited during a high energy condition in a fluvial environment.
\end{abstract}

\section{INTRODUCTION}

Famgbe River is an extension of Nun River called Kolo creek which is within the central sector of the Niger Delta and lies between latitude $4^{0} 5^{0} \mathrm{~N}$ and longitude $6^{0} 5^{\prime}-7^{0} \mathrm{E}$. It is located within the tropical continental margin of the Gulf of Guinea and sub-equatorial region of West Africa. The River has a long course of over 5km and flows in the North-South direction. According to EtuEfeotor(1990), the river flows in the downstream direction flanked to the North east by River Orashi to the Southeast by River Sambero to the West by the River Forcados. The river is tidally affected but increases in volume during the flood season and decrease in volume and current during the dry season. The river takes its course from the River Niger which is about 4100KM long and arises from the Guinea highlands(Allen, 1965). Famgbe River like every other distributaries of the Nun River, supplies water and sediments into the Atlantic Ocean.

Sediments along the beds of Famgbe River are part of the Niger Delta province usually described as theQuaternary deposits. These sediments originated from vast catchment area and geological complex hinterland (Fig.1); they are dispersed through the Delta by rivers, tidal, waves and ocean current (Abam, 2016). 


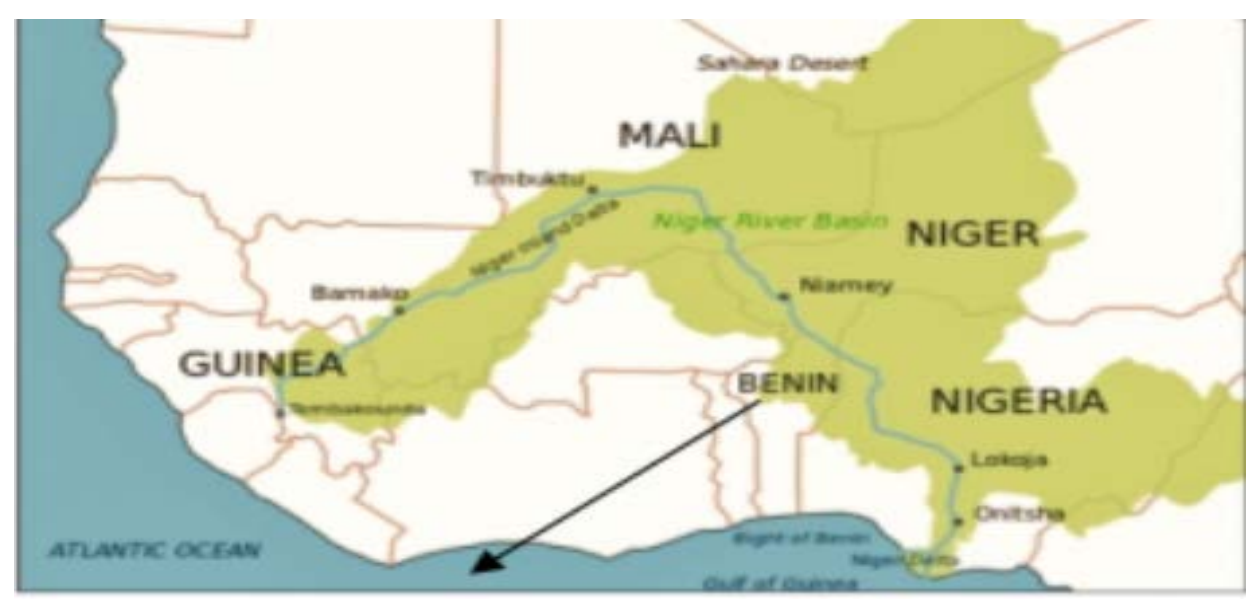

Fig.1. Catchment area of the Niger Delta (Abam, 2016)

These sediments contain clues about source, mode of transportation and environment of deposit which are linked by sedimentary process. Unfortunately, these near surface and shallow Quaternary cover appears not to have received much attention. For this reason the sedimentological study of this Quaternary deposit along the bed of Famgbe River was carried out to determine variation of sedimentological characteristics. The analysis of these sediments will be represented in the form of tables, histograms, frequency curves and as well as statistical data.

\section{TOPOGRAPHY OF THE STUDY AREA}

The study area is mostly characterized by lowlands, as low as $20 \mathrm{~m}$ below sea level. It tends to slope gently in a north-west direction. The area being an extension of the Nun River is drained into the Atlantic Ocean. The Bank of Famgbe River is usually flooded during the wet season

The area lies within the subequatorial region of Nigeria. It is characterized by two major seasons, wet and dry (Iloeje,1979). The wet season begins in March and ends in October, with peak in June and July. There is commonly a period of little or no rain in August while the dry season which is short starts from November and ends in early March. Annual mean rainfall in the area is estimated to be over $3000 \mathrm{~mm}$ (Ojo et al, 1992). The study area is characterized by high temperature and humidity as is common with humid tropical climate. Average annual temperature in the area is about $27^{0}$ (Inyang, 1975), with maximum values in the months of March and April while the lowest temperature occurs in July and August (Amali et al., 1985). The climatic conditions have an intimate relationship with vegetation type in the Area. Due to the high rainfall and humidity which promotes thick vegetation the term tropical rainforest is used to describe the vegetation.

\section{LOCATION AND ACCESSIBILITY}

The study area (Famgbe River) is an extension of River Nun within latitude $4^{0} 5^{0} \mathrm{~N}$ and longitude $6^{0} 5^{\prime}-7^{0} \mathrm{E}$ in Yenagoa local government area Bayelsa state. The area is accessible through land and water transportation system from Yenagoa. 


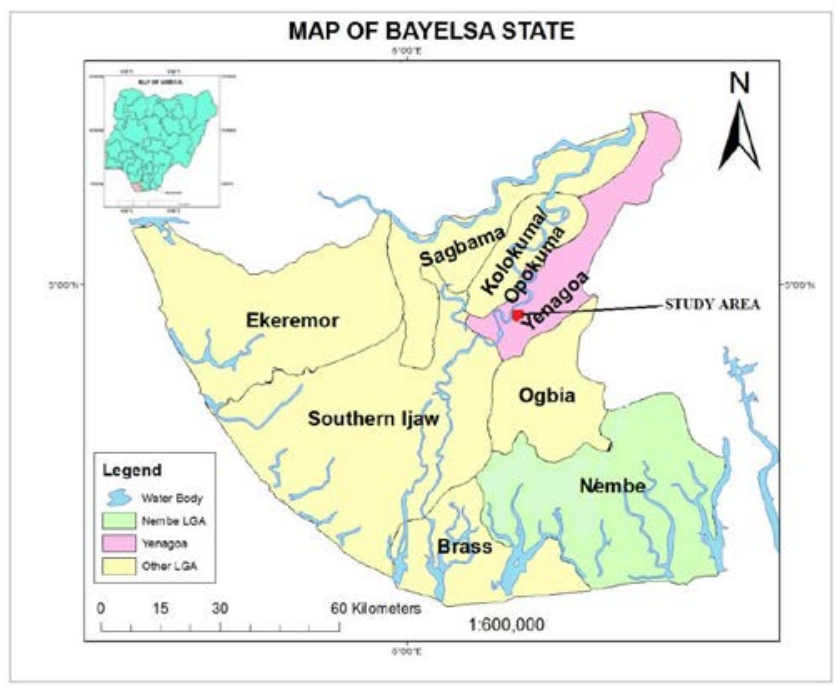

Fig. 2. Map showing the location of study area

\section{GEOMORPHPLOGY OF STUDY AREA}

Recent studies carried by Akpokodje et al., (2014) within the Niger Delta have recognized five major geomorphologic units namely:

- Active and abandoned coastal beaches

- Salt water mangrove swamps

- Freshwater swamps and meander belt

- Sombreiro Warri plain, dry deltaic plain with abundant swamp zones.

- Dry flat land plain

These five geomorphologic units have also been recognized by Short and Stauble (1967) and were divided into three main environments namely continental, transitional and marine environments.

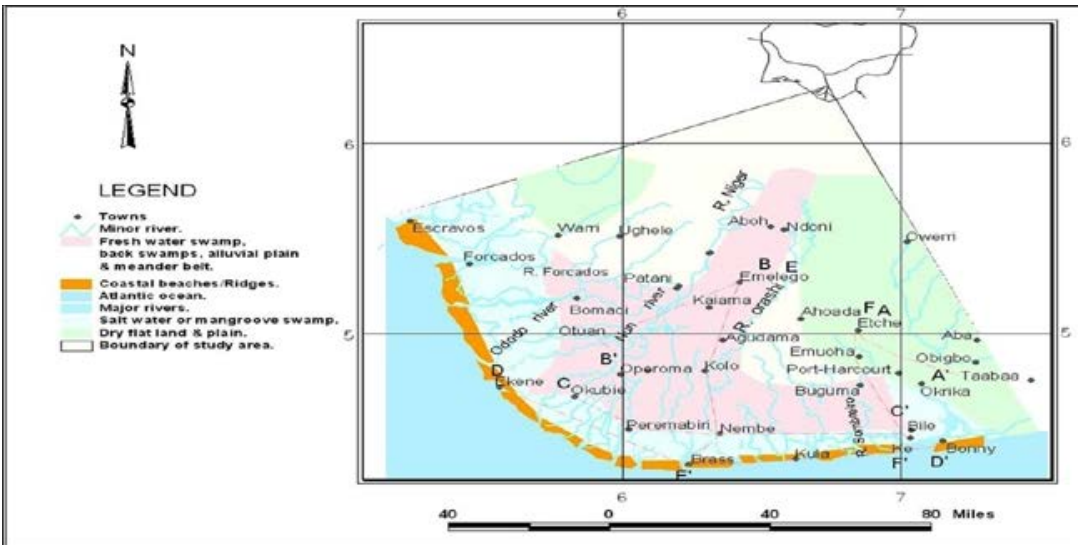

Fig. 3. Geologic map of the Niger Delta (After Akpokodje et al., 2014)

\section{REGIONAL GEOLOGY OF NIGER DELTA}

The study area falls within the Niger Delta basin of the sedimentary part of the geology of Nigeria. The Niger Delta Basin is situated in the Gulf of Guinea in Guinea in equatorial West Africa, between latitudes $3^{\circ} \mathrm{N}$ and $6^{\circ} \mathrm{N}$ and longitudes $5^{\circ} \mathrm{E}$ and $8^{\circ} \mathrm{E}$ (Reijers et al, 1996). The Niger Delta 
is framed on the northwest by a subsurface continuation of the West African Shield, the Benin Flank. The eastern edge of the basin coincides with the Calabar Flank to the south of the Oban Masif (Murat, 1972). The Niger Delta province is a large arcuate wave and tide dominated delta, with sediments ranging in age from Eocene in the north to Quaternary in the south with a thickness of over $12000 \mathrm{~m}$ and area of $7500 \mathrm{~km}^{2}$ (Murat, 1972).

The tectonic evolution of the Niger Delta basin is related to the origin of the Benue Trough which is associated to the splitting of the Gondwanna super continent in the Late Jurassic to Cretaceous times. The Trough itself represents a failed arm (an aulacogen) of the RRR triple junction. As rifting diminished the primary deformational processes; Shale mobility resulted into internal deformation and occurred in response to two processes (Doust, 1989). First, shale diapers formed from loading of poorly compacted, over pressured, prodelta and delta slope clays (Akata formation) by higher density delta front sands (Agbada formation). Then slope instability occurred due to lack of lateral basin ward support for the under compacted delta slope clays. Gravity tectonics ended before deposition of Benin formation and are expressed in complex structures including shale diapers, roll over anticlines, collapsed growth fault crest, back to back features and steeply dipping closely spaced fault faults ( Evamy et al., 1978).

\section{STRATIGRAPY OF THE NIGER DELTA}

The stratigraphy history of the Niger Delta starts alongside the stratigraphic history of the Southeastern Nigerian sedimentary basins. According to Short and Stauble (1967) the Tertiary Niger Delta basin is a sedimentary structure formed as a complex regressive off lap sequence of clastic sediments ranging in thickness from $9000-12000 \mathrm{~m}$. The basin is divided into three major diachronous formations ranging from Eocene to recent base on environmental influence. These environments are continental, paralic and marine environments which are represented by the Akata, Agbada and Benin formation(Table 2.0) respectively. The three major formations are discussed below from the oldest to the youngest.

\section{AKATA FORMATION}

This is a marine pro-delta megafacies of the Tertiary Niger Delta. It underlines the Agbada formation. Consequently, it is dominated by shales and its more than 1380m thick. Its lithological composition comprises of sandstone lenses at the top with uniform grey sand-silty shale at the base. It is rich in microfossils such as forams(Kogbe,1976) indicating shallow marine environment. It ranges from Paleocene to recent in age.

\section{AGBADA FORMATION}

This is a paralic delta front megafacies, which underlied the Benin formation. It consist of an interbedded sequence of sand and shales about 3150m thick on the average. This formation covers the subsurface of the entire delta and has its lateral equivalence as the Ogwashi- Asaba formation (Assez, 1970). It is characterized by transgressive marine barrier foot deposits and tidal channel deposits. The grain sizes are mostly coarse with poor sorting which probably indicates a fluvial origin (Kogbe, 1976). All the hydrocarbon reserves of the Niger Delta accumulate in the sands of this formation. 


\section{BENIN FORMATION}

This is a continental megafacies that overlies the Agbada formation. The Benin formation consist of Late Eocene to recent deposits of alluvial and upper coastal plain deposits that are up to $2000 \mathrm{~m}$ thick (Avbovbo, 1978) unconsolidated sands with thin clay and lignite interbeds. It is assumed to be major aquiferous formation within the Niger Delta region.

Table 1: Geologic units of the Niger Delta (After Akpokodje 1987)

\begin{tabular}{|l|l|l|}
\hline AGE & GEOLOGIC UNIT & LITHOLOGY \\
\hline & Alluvium & Gravel, sand, clay, silt \\
\hline Quaternary & $\begin{array}{l}\text { Freshwater, Backswamp } \\
\text { meander belt }\end{array}$ & Sand, clay, some silt,gravel \\
\hline & $\begin{array}{l}\text { Saltwater, Mangrove swamp } \\
\text { and Backswamp }\end{array}$ & $\begin{array}{l}\text { Medium-Fine sand, clay and } \\
\text { some silt }\end{array}$ \\
\hline $\begin{array}{l}\text { Active/abandoned Beach } \\
\text { ridges }\end{array}$ & Sand, clay and silt \\
\hline Miocene & Sambrerio-Warri Deltaic plain & Sand, clay and silt \\
\hline Eocene & Benin Formation & $\begin{array}{l}\text { Coastal medium sand; } \\
\text { subordinate silt and clay } \\
\text { lenses }\end{array}$ \\
\hline Paleocene & Agbada Formation & Mixture of clay sand and silt \\
\hline
\end{tabular}

\section{METHODOLOGY AND MATERIALS}

\section{FIELD TECHNIQUE}

This basically involves the methods, materials and sampling technique used for the study. The method used in collecting the samples is the transverse collection method. Using the transverse method with the aid of a boat, ten (10) samples were collected along the course of the river bank.. Materials used for sample collection includes:

- Sample bags: These are small polythene bags used to collect and store the samples obtained

- A GPS (Global positioning system): Used to get the coordinates of each location.

- A pail: Used to collect the samples from the river bank

- Masking tape and pen: Used for labeling the samples of various location.

The samples collected from the field were carefully packed and transfer to the laboratory for analysis.

\section{LABORATORY ANALYSIS}

This part of the study was carried out in the laboratory. This involves grain size analysis (sieve analysis) of the samples collected in the field. This deals with the textural analysis of sediments. It is the most important parameter of clastic sediments because it is used in describing the nature of the sediments, agent of transportation, deposition, environmental conditions at the time of deposition, porosity, permeability and classification of the clastic sediments. Two type of method are used for grain size analysis, wet and dry method but for the purpose of this research the dry method was used (Folk and Ward, 1957). 


\section{RESULTS AND DISCUSSIONS}

\section{PRESENTATION OF RESULT}

The sieve analysis was used to determine the grain size characterization of the sediments collected from the study area. The sieve analysis result is presented below.

Histogram plot and cumulative frequency curves for each sample is being presented.

Table 2: Sieve analysis values for Sample 1(Fagmbe River)

LOCATION: 1

\begin{tabular}{|l|l|l|l|l|l|l|}
\hline \multicolumn{2}{|l|}{ Class interval } & Weight \\
$\begin{array}{l}\text { Metric } \\
\text { mm }\end{array}$ & Phi 0 & $\begin{array}{l}\text { Corrected } \\
\text { weight } \\
\text { (gm.) }\end{array}$ & $\begin{array}{l}\text { Cumulati } \\
\text { ve weight } \\
\text { (gm, }\end{array}$ & $\begin{array}{l}\text { Correcte } \\
\text { d weight } \\
\text { (\%) }\end{array}$ & $\begin{array}{l}\text { Cumulativ } \\
\text { e weight } \\
\text { (\%) }\end{array}$ \\
\hline$>2$ & -2 to -1 & 0.00 & 2.99 & 2.99 & 1.56 & 1.56 \\
\hline $2-1$ & -1 to 0 & 0.33 & 3.32 & 6.31 & 1.73 & 3.29 \\
\hline $1-0.5$ & 0 to 1 & 3.47 & 6.46 & 12.77 & 3.37 & 6.66 \\
\hline $0.5-0.25$ & 1 to 2 & 124.41 & 127.4 & 140.17 & 66.57 & 72.23 \\
\hline $\begin{array}{l}0.25- \\
0.125\end{array}$ & 2 to 3 & 41.12 & 44.11 & 184.28 & 23.04 & 95.27 \\
\hline $\begin{array}{l}0.125- \\
0.63\end{array}$ & 3 to 4 & 0.51 & 3.5 & 187.78 & 1.82 & 97.09 \\
\hline$<0.0$ & $>4$ & 0.61 & 3.5 & 191.38 & 1.88 & 98.87 \\
\hline $\begin{array}{l}\text { Total } \\
\text { weight }\end{array}$ & & 170.45 & & & & \\
\hline
\end{tabular}

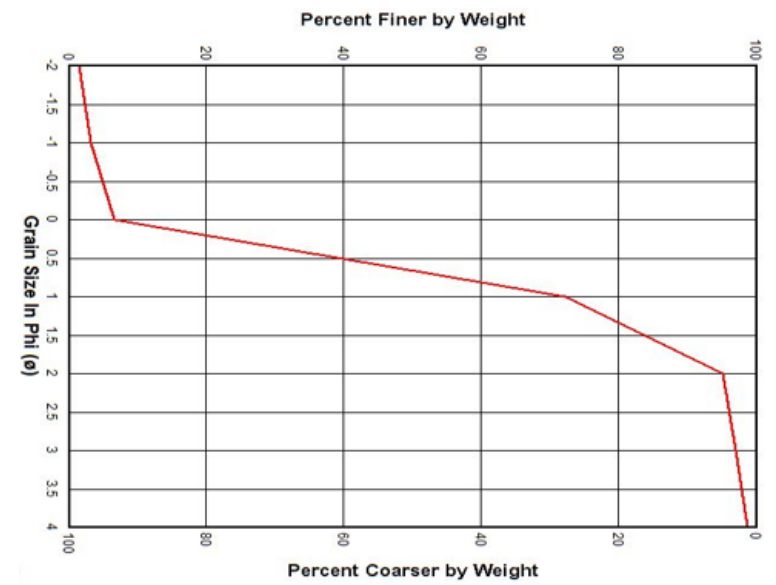

Fig. 4. Cumulative frequency curve for sample 1 
Table 3: Sieve analysis values for Sample 2 (Fagmbe River)

LOCATION 2

\begin{tabular}{|c|c|c|c|c|c|c|}
\hline \multicolumn{2}{|c|}{ Class interval } & \multirow{2}{*}{$\begin{array}{l}\text { Weight } \\
\text { retained }\end{array}$} & \multirow{2}{*}{$\begin{array}{l}\text { Corrected } \\
\text { weight } \\
\text { (gm.) }\end{array}$} & \multirow{2}{*}{$\begin{array}{l}\text { Cumulative } \\
\text { weight } \\
\text { (gm,) }\end{array}$} & \multirow{2}{*}{$\begin{array}{l}\text { Corrected } \\
\text { weight } \\
(\%)\end{array}$} & \multirow{2}{*}{$\begin{array}{l}\text { Cumulative } \\
\text { weight (\%) }\end{array}$} \\
\hline Metric mm & Phi 0 & & & & & \\
\hline$>2$ & -2 to -1 & 0.08 & 0.12 & 0.12 & 0.08 & 0.08 \\
\hline $2-1$ & -1 to 0 & 0.19 & 0.23 & 0.35 & 0.16 & 0.24 \\
\hline $1-0.5$ & 0 to 1 & 16.59 & 16.63 & 16.98 & 11.41 & 11.65 \\
\hline $0.5-0.25$ & 1 to 2 & 111.02 & 111.06 & 128.04 & 76.23 & 87.88 \\
\hline $0.25-0.125$ & 2 to 3 & 16.57 & 16.61 & 144.65 & 11.40 & 99.28 \\
\hline $\begin{array}{c}0.125- \\
0.63\end{array}$ & 3 to 4 & 0.58 & 0.62 & 145.27 & 0.43 & 99.71 \\
\hline$<0.0$ & $>4$ & 0.37 & 0.41 & 145.68 & 0.28 & 99.99 \\
\hline $\begin{array}{c}\text { Total } \\
\text { weight }\end{array}$ & & 144.24 & & & & \\
\hline
\end{tabular}

Percent Finer by Weight

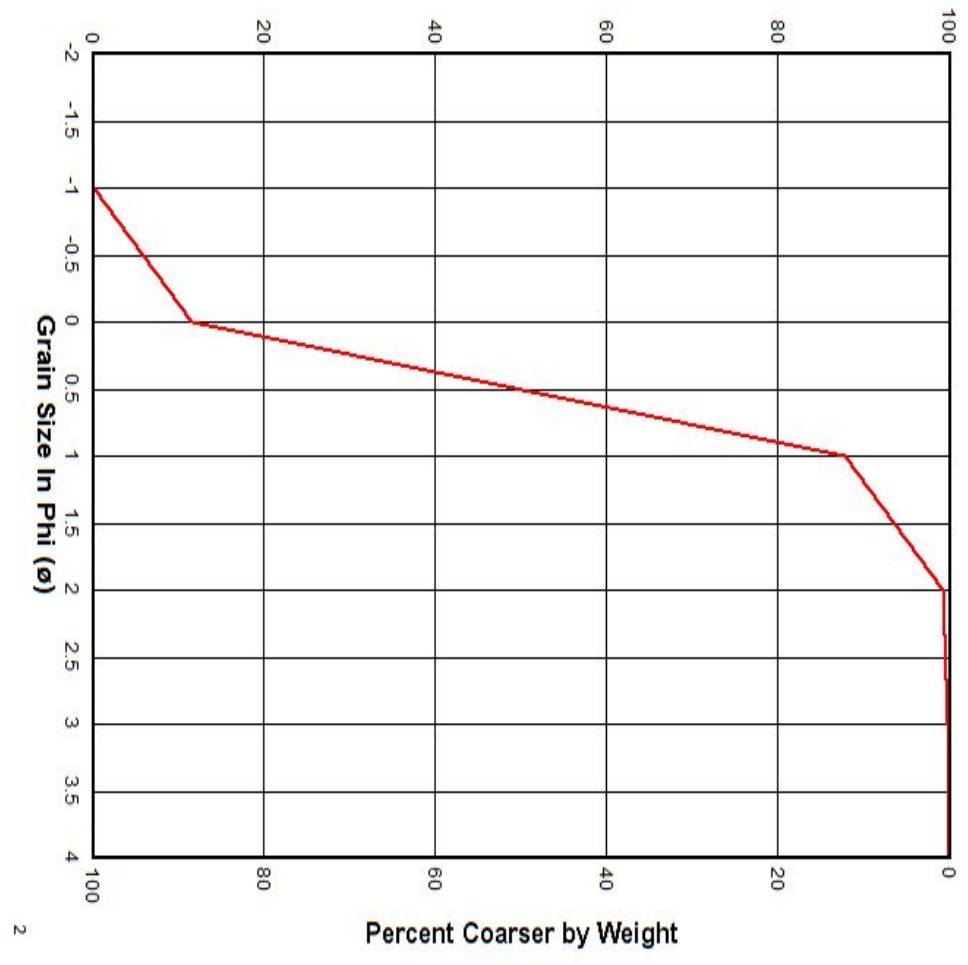

Fig. 5. Cumulative frequency curve for sample 2 (Famgbe River) 
Table 4: Sieve analysis values for Sample 3 (Fagmbe River)

LOCATION 3

\begin{tabular}{|l|l|l|l|l|l|l|}
\hline \multicolumn{2}{|l|}{ Class interval } & \multirow{2}{*}{$\begin{array}{l}\text { Weight } \\
\text { retained }\end{array}$} & $\begin{array}{l}\text { Corrected } \\
\text { weight (gm.) }\end{array}$ & $\begin{array}{l}\text { Cumulative } \\
\text { weight (gm,) }\end{array}$ & $\begin{array}{l}\text { Corrected } \\
\text { weight (\%) }\end{array}$ & $\begin{array}{l}\text { Cumulative } \\
\text { weight (\%) }\end{array}$ \\
\cline { 1 - 2 } Metric mm & Phi 0 & & & & & \\
\cline { 1 - 5 }$>2$ & -2 to -1 & 0.00 & 0.48 & 0.48 & 0.28 & 0.28 \\
\hline $2-1$ & -1 to 0 & 0.51 & 0.99 & 1.47 & 0.87 & 1.15 \\
\hline $1-0.5$ & 0 to 1 & 12.12 & 12.6 & 14.07 & 7.45 & 8.6 \\
\hline $0.5-0.25$ & 1 to 2 & 115.5 & 115.98 & 130.05 & 68.93 & 77.53 \\
\hline $0.25-0.125$ & 2 to 3 & 33.09 & 33.57 & 163.62 & 19.95 & 97.48 \\
\hline $0.125-0.63$ & 3 to 4 & 1.73 & 2.21 & 165.83 & 1.31 & 98.79 \\
\hline$<0.0$ & $>4$ & 1.93 & 2.41 & 168.24 & 1.43 & 100.00 \\
\hline Total weight & & 164.88 & & & & \\
\hline
\end{tabular}

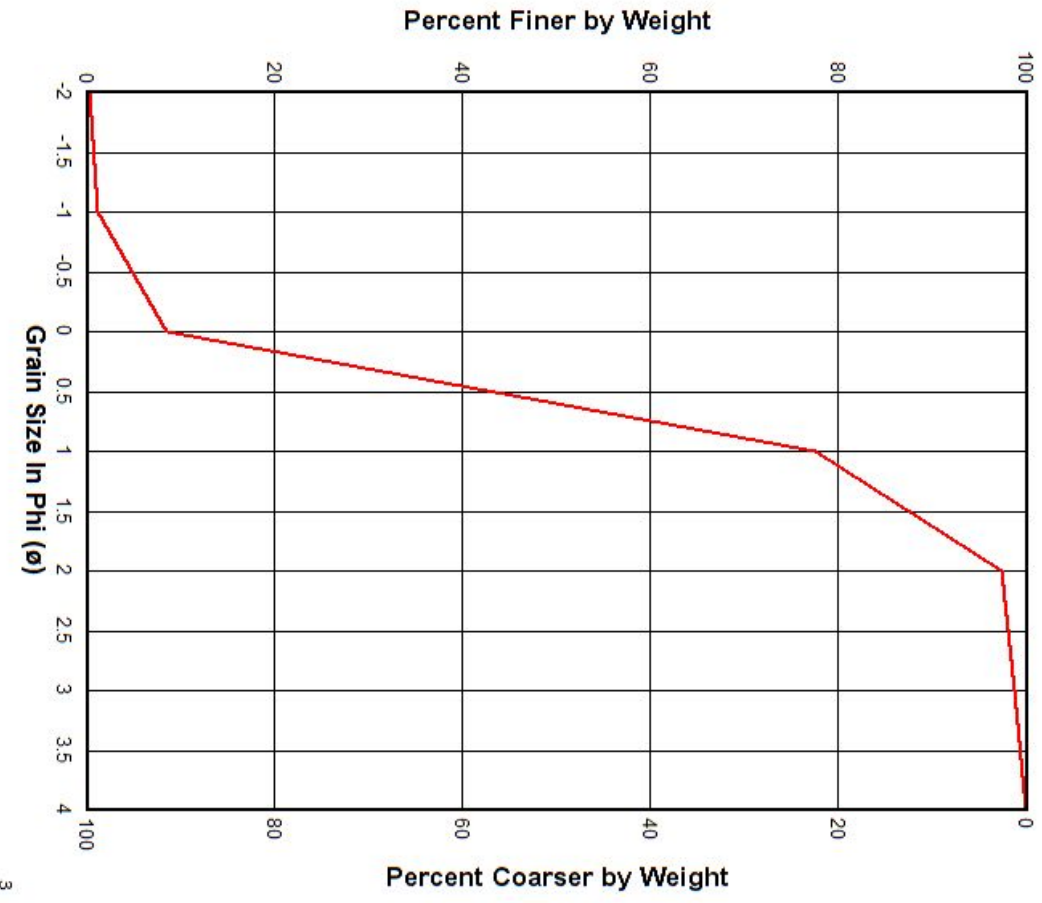

Fig. 6. Cumulative frequency curve for sample 3 
Table 5: Sieve analysis results of sample 4 (Fagmbe River)

LOCATION 4

\begin{tabular}{|c|c|c|c|c|c|c|}
\hline \multicolumn{2}{|c|}{ Class interval } & \multirow{2}{*}{$\begin{array}{l}\text { Weight } \\
\text { retained }\end{array}$} & \multirow{2}{*}{$\begin{array}{l}\text { Corrected } \\
\text { weight } \\
\text { (gm.) }\end{array}$} & \multirow{2}{*}{$\begin{array}{l}\text { Cumulativ } \\
\text { e weight } \\
\text { (gm,) }\end{array}$} & \multirow{2}{*}{$\begin{array}{l}\text { Correcte } \\
\text { d weight } \\
(\%)\end{array}$} & \multirow{2}{*}{$\begin{array}{l}\text { Cumulative } \\
\text { weight (\%) }\end{array}$} \\
\hline $\begin{array}{l}\text { Metric } \\
\text { mm }\end{array}$ & Phi $\omega$ & & & & & \\
\hline$>2$ & -2 to -1 & 0.00 & 0.37 & 0.37 & 0.27 & 0.27 \\
\hline $2-1$ & -1 to 0 & 0.03 & 0.40 & 0.77 & 0.29 & 0.56 \\
\hline $1-0.5$ & 0 to 1 & 5.07 & 5.44 & 6.21 & 3.96 & 4.52 \\
\hline $0.5-0.25$ & 1 to 2 & 96.92 & 97.29 & 103.5 & 70.88 & 75.4 \\
\hline $0.25-0.125$ & 2 to 3 & 31.83 & 32.20 & 135.7 & 23.46 & 98.86 \\
\hline $\begin{array}{l}0.125- \\
0.63\end{array}$ & 3 to 4 & 0.47 & 0.84 & 136.54 & 0.61 & 99.47 \\
\hline$<0.0$ & $>4$ & 0.34 & 0.71 & 137.25 & 0.52 & 99.99 \\
\hline $\begin{array}{l}\text { Total } \\
\text { weight }\end{array}$ & & 134.66 & & & & \\
\hline
\end{tabular}

Percent Finer by Weight

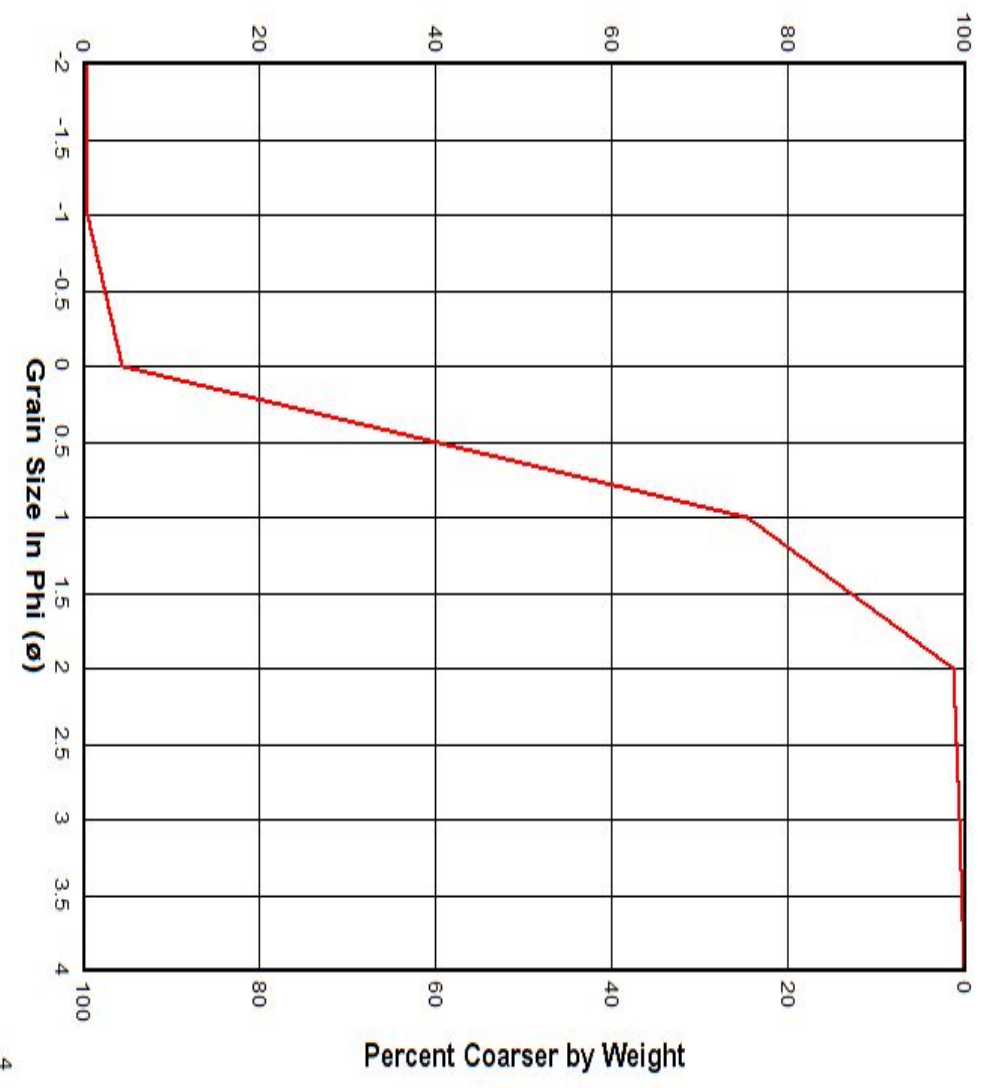

Fig. 7. Cumulative frequency curve for sample 4 (Fambge River) 
Table 6: Sieve analysis value of sample 5(Fagmbe River)

LOCATION 5

\begin{tabular}{|c|c|c|c|c|c|c|}
\hline \multicolumn{2}{|c|}{ Class interval } & \multirow{2}{*}{$\begin{array}{l}\text { Weight } \\
\text { retained }\end{array}$} & \multirow{2}{*}{$\begin{array}{l}\text { Corrected } \\
\text { weight } \\
\text { (gm.) }\end{array}$} & \multirow{2}{*}{$\begin{array}{l}\text { Cumulativ } \\
\text { e weight } \\
\text { (gm,) }\end{array}$} & \multirow{2}{*}{$\begin{array}{l}\text { Correcte } \\
\text { d weight } \\
(\%)\end{array}$} & \multirow{2}{*}{$\begin{array}{l}\text { Cumulativ } \\
\text { e weight } \\
(\%)\end{array}$} \\
\hline $\begin{array}{l}\text { Metric } \\
\mathrm{mm}\end{array}$ & Phi $\oplus$ & & & & & \\
\hline$>2$ & -2 to -1 & 0.1 & 1.26 & 1.26 & 0.91 & 0.91 \\
\hline $2-1$ & -1 to 0 & 0.56 & 1.72 & 2.98 & 1.24 & 2.15 \\
\hline $1-0.5$ & 0 to 1 & 19.59 & 20.75 & 23.73 & 15.03 & 17.18 \\
\hline $0.5-0.25$ & 1 to 2 & 86.73 & 87.89 & 111.62 & 63.70 & 80.88 \\
\hline $0.25-0.125$ & 2 to 3 & 22 & 23.16 & 134.78 & 16.79 & 97.67 \\
\hline $\begin{array}{l}0.125- \\
0.63\end{array}$ & 3 to 4 & 0.38 & 1.54 & 136.32 & 1.11 & 98.78 \\
\hline$<0.0$ & $>4$ & 0.49 & 1.65 & 137.97 & 1.19 & 99.97 \\
\hline $\begin{array}{l}\text { Total } \\
\text { weight }\end{array}$ & & 129.85 & & & & \\
\hline
\end{tabular}

Percent Finer by Weight

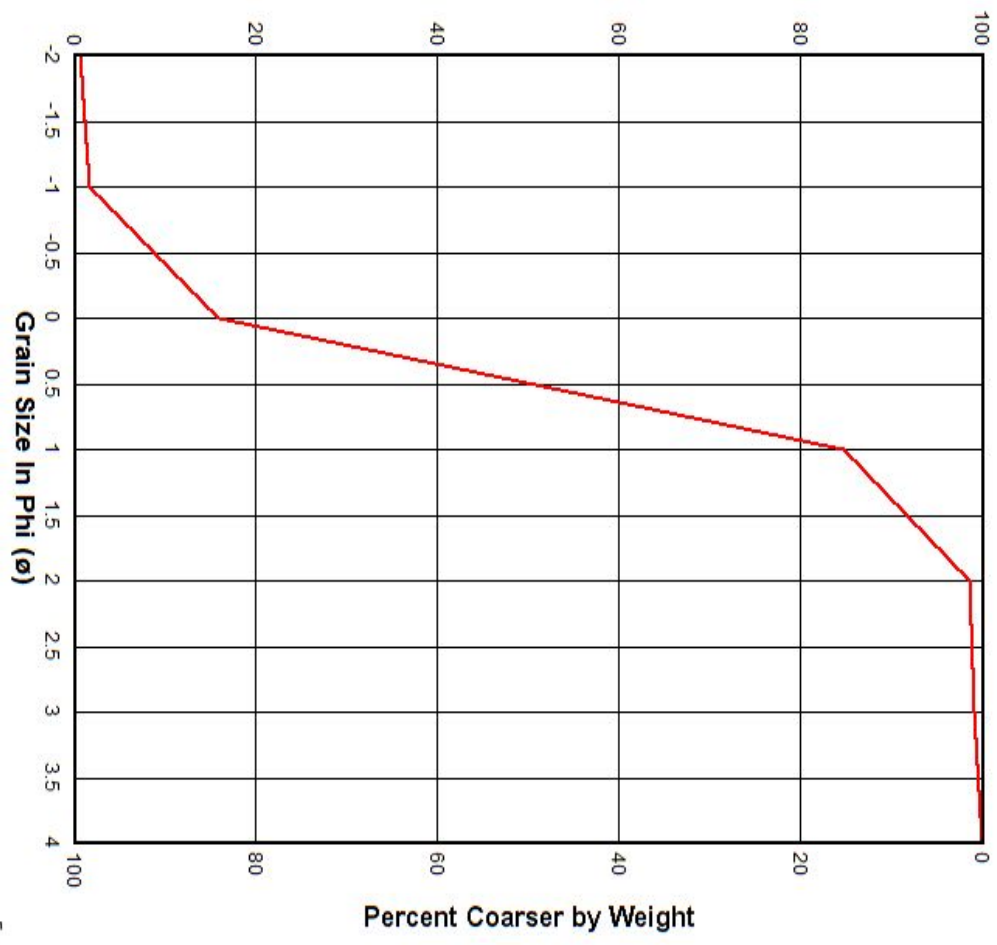

Fig. 8. Cumulative frequency curve for sample 5 
LOCATION 6

Table 7: Sieve analysis values of sample 6(Fagmbe River)

\begin{tabular}{|l|l|l|l|l|l|l|}
\hline \multicolumn{2}{|l|}{ Class interval } & $\begin{array}{l}\text { Weight } \\
\text { retained }\end{array}$ & $\begin{array}{l}\text { Corrected } \\
\text { weight } \\
\text { (gm.) }\end{array}$ & $\begin{array}{l}\text { Cumulativ } \\
\text { e weight } \\
\text { (gm, })\end{array}$ & $\begin{array}{l}\text { Corrected } \\
\text { weight } \\
\text { (\%) }\end{array}$ & $\begin{array}{l}\text { Cumulative } \\
\text { weight (\%) }\end{array}$ \\
\hline$>2$ & -2 to -1 & 0.14 & 0.21 & 0.21 & 0.67 & 0.67 \\
\hline $2-1$ & -1 to 0 & 0.68 & 1.75 & 2.96 & 0.97 & 1.64 \\
\hline $1-0.5$ & 0 to 1 & 24.58 & 25.65 & 28.61 & 14.20 & 15.84 \\
\hline $0.5-0.25$ & 1 to 2 & 123.47 & 124.54 & 153.15 & 68.99 & 84.83 \\
\hline $0.25-0.125$ & 2 to 3 & 23.69 & 24.76 & 177.91 & 13.72 & 98.55 \\
\hline $\begin{array}{l}0.125- \\
0.63\end{array}$ & 3 to 4 & 0.13 & 1.20 & 179.11 & 0.66 & 99.21 \\
\hline$<0.0$ & $>4$ & 0.33 & 1.40 & 180.51 & 0.76 & 99.97 \\
\hline $\begin{array}{l}\text { Total } \\
\text { weight }\end{array}$ & & 173.02 & & & & \\
\hline
\end{tabular}

Percent Finer by Weight

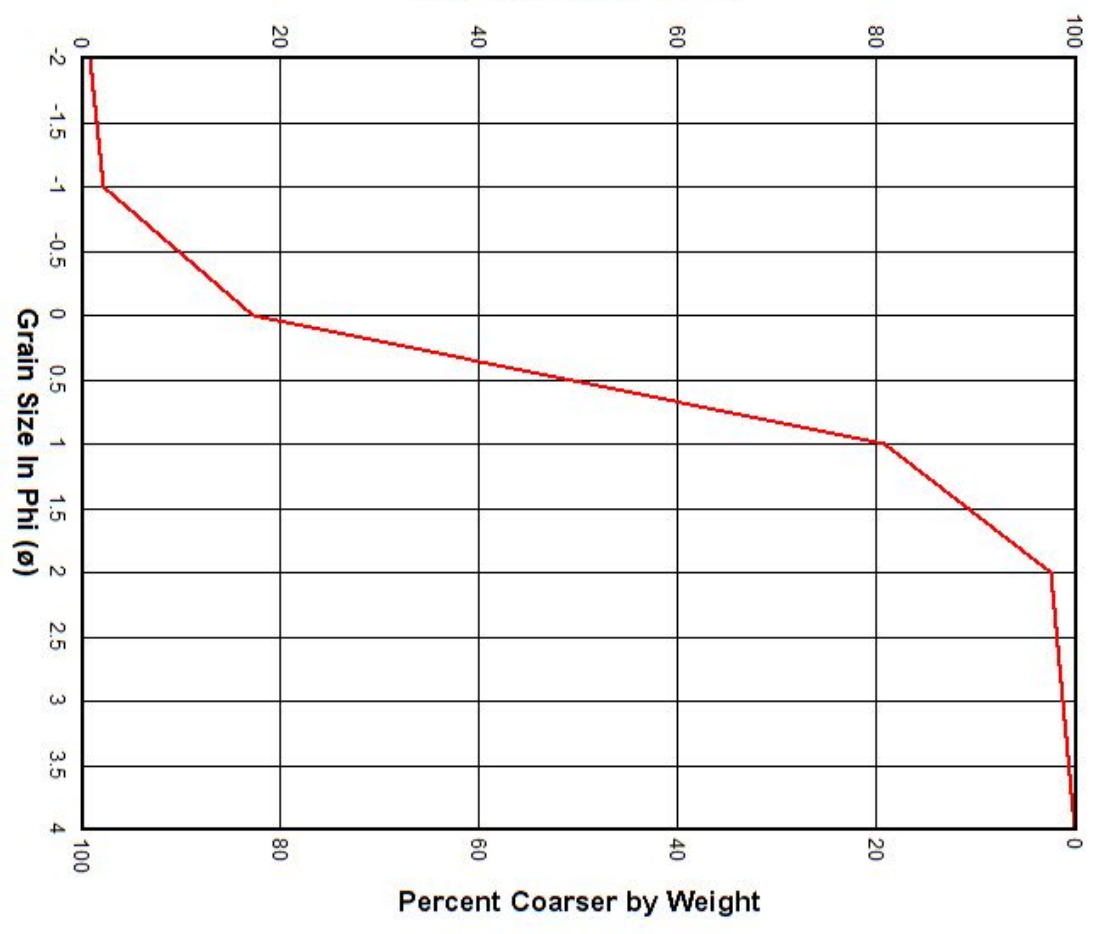

Fig. 9. Cumulative frequency curve for sample 6 (Famgbe River) 
Table 8: Sieve analysis values of sample 7 (Fagmbe River)

LOCATION 7

\begin{tabular}{|c|c|c|c|c|c|c|}
\hline \multicolumn{2}{|c|}{ Class interval } & \multirow{2}{*}{$\begin{array}{l}\text { Weight } \\
\text { retained }\end{array}$} & \multirow{2}{*}{$\begin{array}{l}\text { Corrected } \\
\text { weight } \\
\text { (gm.) }\end{array}$} & \multirow{2}{*}{$\begin{array}{l}\text { Cumulati } \\
\text { ve weight } \\
\text { (gm,) }\end{array}$} & \multirow{2}{*}{$\begin{array}{l}\text { Correcte } \\
\text { d weight } \\
\text { (\%) }\end{array}$} & \multirow{2}{*}{$\begin{array}{l}\text { Cumulativ } \\
\text { e weight } \\
\text { (\%) }\end{array}$} \\
\hline $\begin{array}{l}\text { Metric } \\
\text { mm }\end{array}$ & Phi 0 & & & & & \\
\hline$>2$ & -2 to -1 & 0.03 & 0.85 & 0.85 & 0.50 & 0.50 \\
\hline $2-1$ & -1 to 0 & 0.41 & 1.23 & 2.08 & 0.72 & 1.22 \\
\hline $1-0.5$ & 0 to 1 & 25.02 & 25.84 & 27.92 & 15.13 & 16.35 \\
\hline $0.5-0.25$ & 1 to 2 & 117.55 & 118.37 & 146.29 & 69.33 & 85.68 \\
\hline $\begin{array}{l}0.25- \\
0.125\end{array}$ & 2 to 3 & 20.91 & 21.73 & 168.02 & 12.72 & 98.40 \\
\hline $\begin{array}{l}0.125- \\
0.63\end{array}$ & 3 to 4 & 0.1 & 0.92 & 168.94 & 0.53 & 98.93 \\
\hline$<0.0$ & $>4$ & 0.97 & 1.79 & 170.73 & 1.04 & 99.97 \\
\hline $\begin{array}{l}\text { Total } \\
\text { weight }\end{array}$ & & 164.99 & & & & \\
\hline
\end{tabular}

Percent Finer by Weight

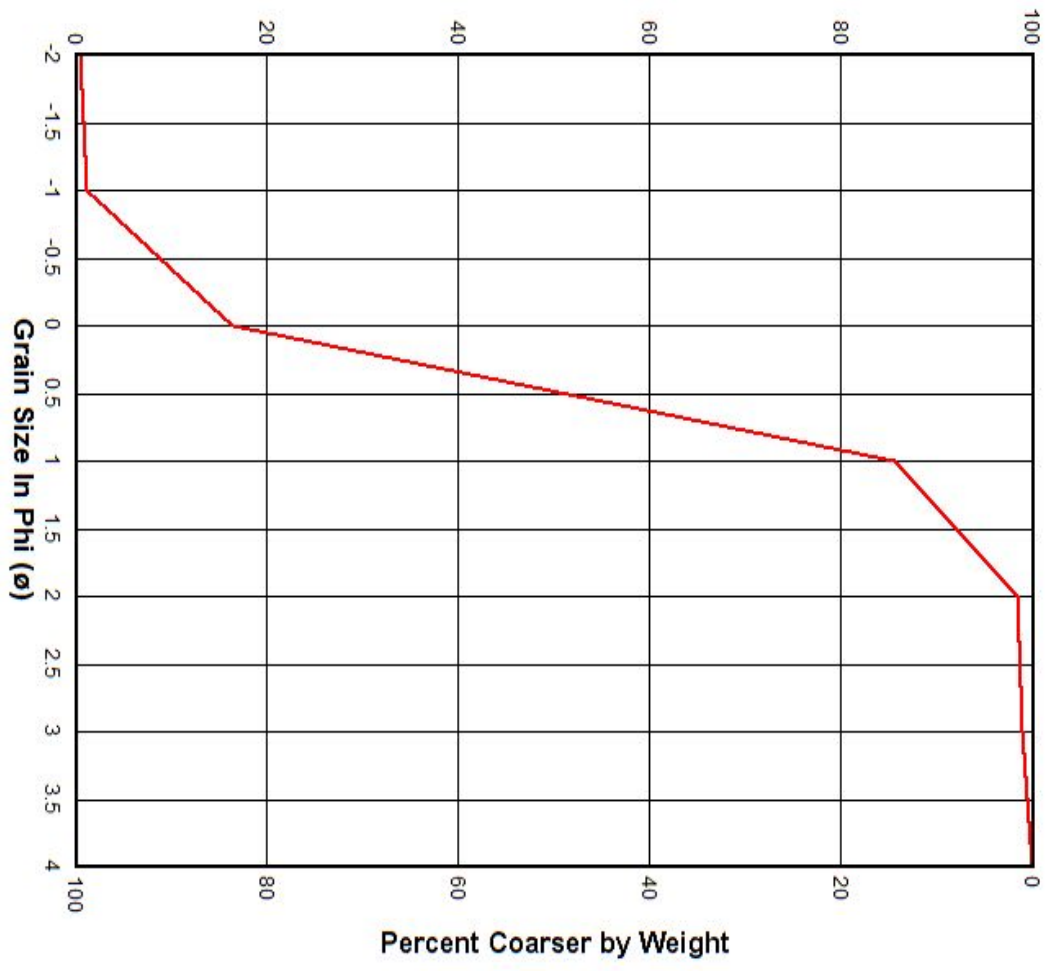

Fig. 10. Cumulative frequency curve for sample 7 
Table 9: Sieve analysis values of sample 8 (Fagmbe River)

LOCATION: 8

\begin{tabular}{|c|c|c|c|c|c|c|}
\hline \multicolumn{2}{|c|}{ Class interval } & \multirow{2}{*}{$\begin{array}{l}\text { Weig } \\
\text { ht } \\
\text { retain } \\
\text { ed }\end{array}$} & \multirow{2}{*}{$\begin{array}{l}\text { Correcte } \\
\text { d weight } \\
\text { (gm.) }\end{array}$} & \multirow{2}{*}{$\begin{array}{l}\text { Cumula } \\
\text { tive } \\
\text { weight } \\
\text { (gm,) }\end{array}$} & \multirow{2}{*}{$\begin{array}{l}\text { Correcte } \\
\text { d weight } \\
(\%)\end{array}$} & \multirow{2}{*}{$\begin{array}{l}\text { Cumulative } \\
\text { weight (\%) }\end{array}$} \\
\hline $\begin{array}{l}\text { Metric } \\
\text { mm }\end{array}$ & Phi 0 & & & & & \\
\hline$>2$ & -2 to -1 & 0.01 & 22.75 & 22.75 & 6.22 & 6.22 \\
\hline $2-1$ & -1 to 0 & 0.22 & 22.96 & 45.71 & 6.30 & 12.52 \\
\hline $1-0.5$ & 0 to 1 & 15.6 & 38.34 & 84.05 & 10.50 & 23.02 \\
\hline $0.5-0.25$ & 1 to 2 & 132.1 & 154.84 & 238.89 & 42.39 & 65.41 \\
\hline $0.25-0.125$ & 2 to 3 & 32.94 & 55.68 & 294.57 & 15.25 & 80.66 \\
\hline $\begin{array}{l}0.125- \\
0.63\end{array}$ & 3 to 4 & 23.45 & 46.19 & 340.76 & 12.65 & 93.31 \\
\hline$<0.0$ & $>4$ & 1.37 & 24.44 & 365.2 & 6.70 & 100.00 \\
\hline $\begin{array}{l}\text { Total } \\
\text { weight }\end{array}$ & & $\begin{array}{l}205.6 \\
9\end{array}$ & & & & \\
\hline
\end{tabular}

Percent Finer by Weight

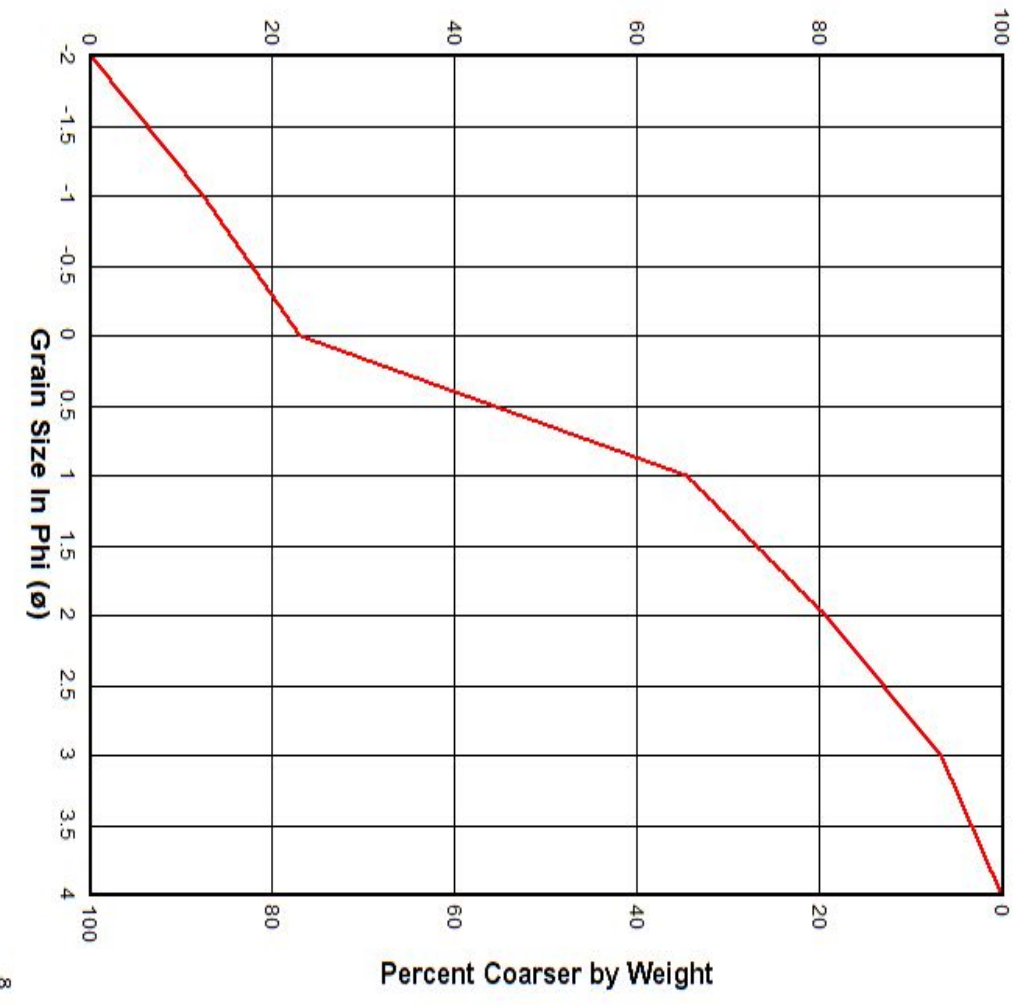

Fig. 11. Cumulative frequency curve for sample 8 (Famgbe River) 
Table 10 Sieve analysis values of sample 9 (Fagmbe River)

LOCATION: 9

\begin{tabular}{|l|l|l|l|l|l|l|}
\hline \multicolumn{2}{|l|}{ Class interval } & $\begin{array}{l}\text { Weigh } \\
\text { t } \\
\text { retaine } \\
\text { d }\end{array}$ & $\begin{array}{l}\text { Corrected } \\
\text { weight } \\
\text { (gm.) }\end{array}$ & $\begin{array}{l}\text { Cumulativ } \\
\text { e weight } \\
\text { (gm, }\end{array}$ & $\begin{array}{l}\text { Correcte } \\
\text { d weight } \\
\text { (\%) }\end{array}$ & $\begin{array}{l}\text { Cumulativ } \\
\text { e weight } \\
\text { (\%) }\end{array}$ \\
\hline$>2$ & $\begin{array}{l}\text { Metric mm } \\
1\end{array}$ & 0.01 & 22.75 & 22.75 & 6.22 & 6.22 \\
\hline $2-1$ & -1 to 0 & 0.22 & 22.96 & 45.71 & 6.30 & 12.52 \\
\hline $1-0.5$ & 0 to 1 & 15.6 & 38.34 & 84.05 & 10.50 & 23.02 \\
\hline $0.5-0.25$ & 1 to 2 & 132.1 & 154.84 & 238.89 & 42.39 & 65.41 \\
\hline $0.25-0.125$ & 2 to 3 & 32.94 & 55.68 & 294.57 & 15.25 & 80.66 \\
\hline $\begin{array}{l}0.125- \\
0.63\end{array}$ & 3 to 4 & 23.45 & 46.19 & 340.76 & 12.65 & 93.31 \\
\hline$<0.0$ & $>4$ & 1.37 & 24.44 & 365.2 & 6.70 & 100 \\
\hline $\begin{array}{l}\text { Total } \\
\text { weight }\end{array}$ & & 205.69 & & & & \\
\hline
\end{tabular}

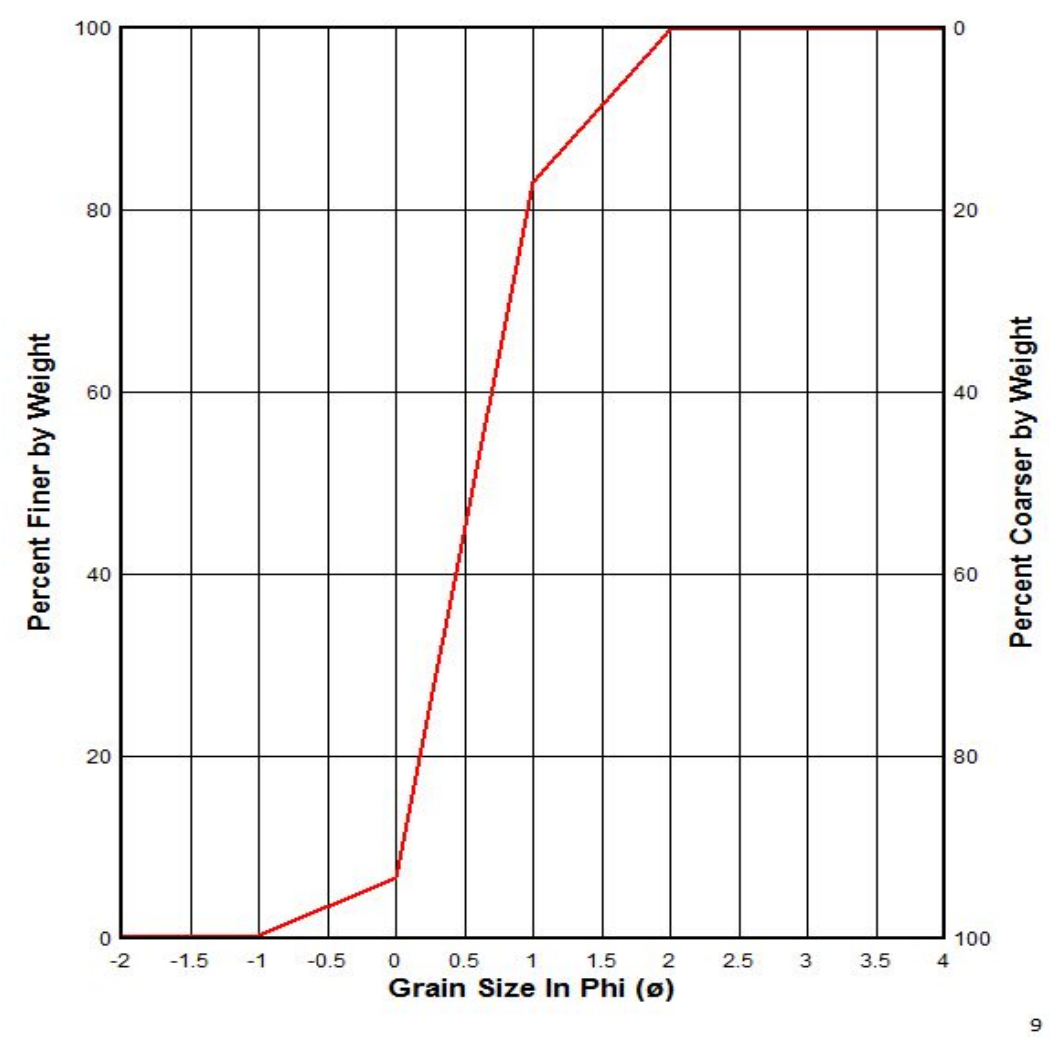

Fig. 12. Cumulative frequency curve for sample 9 
Table 11: Sieve analysis values of sample 10

LOCATION: 10

\begin{tabular}{|c|c|c|c|c|c|c|}
\hline \multicolumn{2}{|c|}{ Class interval } & \multirow{2}{*}{$\begin{array}{l}\text { Weight } \\
\text { retained }\end{array}$} & \multirow{2}{*}{$\begin{array}{l}\text { Corrected } \\
\text { weight } \\
\text { (gm.) }\end{array}$} & \multirow{2}{*}{$\begin{array}{l}\text { Cumulativ } \\
\text { e weight } \\
\text { (gm,) }\end{array}$} & \multirow{2}{*}{$\begin{array}{l}\text { Correcte } \\
\text { d weight } \\
(\%)\end{array}$} & \multirow{2}{*}{$\begin{array}{l}\text { Cumulativ } \\
\text { e weight } \\
\text { (\%) }\end{array}$} \\
\hline $\begin{array}{l}\text { Metric } \\
\text { mm }\end{array}$ & Phi $\oplus$ & & & & & \\
\hline$>2$ & -2 to -1 & 0.02 & 0.28 & 0.28 & 0.16 & 0.16 \\
\hline $2-1$ & -1 to 0 & 0.12 & 0.38 & 0.66 & 0.22 & 0.38 \\
\hline $1-0.5$ & 0 to 1 & 9.74 & 10 & 10.66 & 5.67 & 6.05 \\
\hline $0.5-0.25$ & 1 to 2 & 124.67 & 124.93 & 135.59 & 70.88 & 76.93 \\
\hline $0.25-0.125$ & 2 to 3 & 39.54 & 39.8 & 175.39 & 22.58 & 99.51 \\
\hline $\begin{array}{l}0.125- \\
0.63 \\
\end{array}$ & 3 to 4 & 0.02 & 0.28 & 175.67 & 0.16 & 99.67 \\
\hline$<0.0$ & $>4$ & 0.32 & 0.58 & 176.25 & 0.33 & 100 \\
\hline $\begin{array}{l}\text { Total } \\
\text { weight }\end{array}$ & & 174.23 & & & & \\
\hline
\end{tabular}

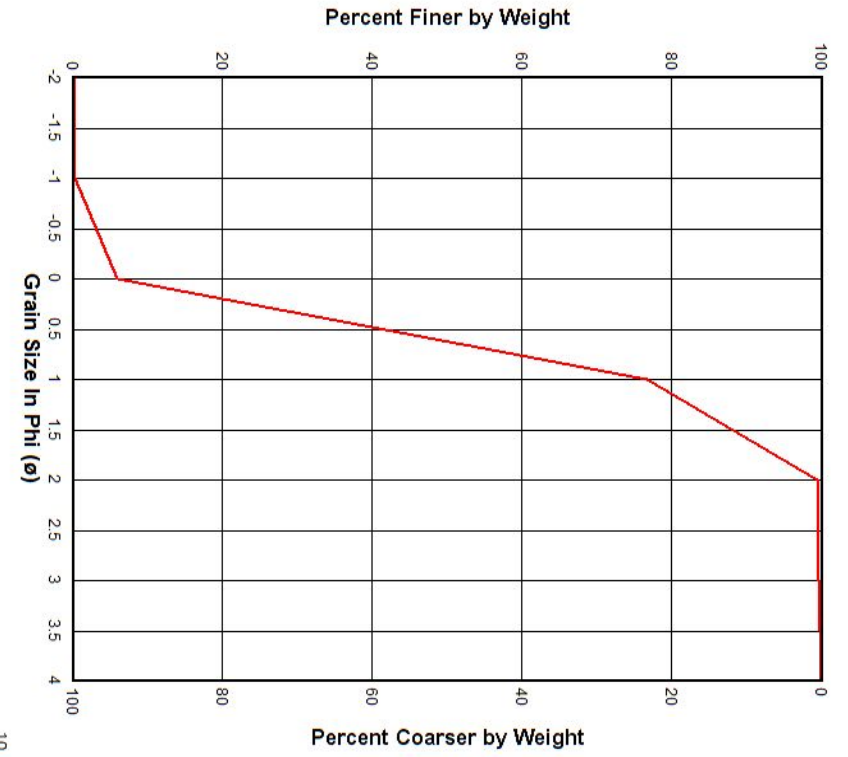


Fig. 13. Cumulative frequency curve for sample 10 (Famgbe River)

\section{DISCUSSION}

Table 12: Summary of statistical parameter and interpretation of Samples from Famgbe River

\begin{tabular}{|c|c|c|c|c|c|}
\hline Sample code & Mean & Sorting & Skewness & Kurtosis & Interpretation \\
\hline 1 & 0.77 & 0.7 & 0.17 & 1.17 & $\begin{array}{c}\text { Coarse sand, Moderately well sorted, } \\
\text { postively skewwed, Leptokurtic }\end{array}$ \\
\hline 2 & 0.49 & 0.56 & 0.02 & 0.14 & $\begin{array}{c}\text { Coarse sand, Moderately well sorted, } \\
\text { postively skewwed, very platykurtic }\end{array}$ \\
\hline 3 & 0.67 & 0.65 & 0.15 & 1.4 & $\begin{array}{c}\text { Coarse sand, Moderately well sorted, } \\
\text { postively skewwed, Leptokurtic }\end{array}$ \\
\hline 4 & 0.72 & 0.57 & 0.25 & 1.05 & $\begin{array}{c}\text { Coarse sand, Moderately well sorted, } \\
\text { postively skewwed, mesokurtic }\end{array}$ \\
\hline 5 & -0.48 & 0.62 & -0.01 & 1.37 & $\begin{array}{c}\text { very Coarse sand, Moderately sorted, nearly } \\
\text { symmetrically skewed, Leptokurtic }\end{array}$ \\
\hline 6 & -0.54 & 0.7 & 0.02 & 1.39 & $\begin{array}{c}\text { very Coarse sand, Moderately sorted, Very } \\
\text { positively skewed, Leptokurtic }\end{array}$ \\
\hline 7 & -0.47 & 0.62 & -0.01 & 1.34 & $\begin{array}{c}\text { very Coarse sand, Moderately sorted, nearly } \\
\text { symmetrically skewed, Leptokurtic }\end{array}$ \\
\hline 8 & -0.74 & 1.48 & 0.08 & 1.26 & $\begin{array}{c}\text { Very Coarse sand, poorly sorted, Nearly } \\
\text { symmetrically skewed, Leptokurtic }\end{array}$ \\
\hline 9 & 0.57 & 0.53 & 0.11 & 1.24 & $\begin{array}{c}\text { Coarse sand, Moderately well sorted, } \\
\text { postively skewwed, Leptokurtic }\end{array}$ \\
\hline 10 & 0.69 & 0.61 & 0.19 & 1.16 & $\begin{array}{c}\text { Coarse sand, Moderately well sorted, } \\
\text { positively skewed, Leptokurtic }\end{array}$ \\
\hline Average & 0.168 & 0.704 & 0.097 & 1.152 & $\begin{array}{c}\text { Coarse sand, Moderately well sorted, } \\
\text { positively skewed, Leptokurtic }\end{array}$ \\
\hline
\end{tabular}

TEXTURAL CHARACTERISTICS

\section{Graphic mean}

The graphic mean ranges from from $-0.74 \phi$ (very coarse grained) to $0.77 \phi$ (coarse grained) with an average of $0.17 \phi$ (coarse grained) which indicates that the sediments are predominantly coarse grained sand. The presence of little or no fine grain indicates that higher energy currents dominate the deposition of the sediments (Ezenwaka, 2015).

\section{Graphic Sorting}

The graphic standard deviation ranges from $1.48 \phi$ (poorly sorted) $-0.53 \phi$ (moderately well sorted) with an average of $0.70 \phi$ (moderately well sorted) which indicates that the sediments are dominantly well sorted. The dominant moderately well sorted character infers that the transportation responsible for the deposition was very turbulent where there is a lot of winnowing (Shettima, et al., 2016). This suggests that the prevalence of strong energy conditions within the time of deposition. 


\section{Graphic Skewness}

The values of inclusive graphic skewness ranging from $-0.01 \phi$ (nearly symmetrical) to $0.25 \phi$ (positively skewed) with an average of $0.09 \phi$ (positively skewed) which indicates that the sediments are dominantly positively skewed in nature. This suggests that the coarse admixture exceeds the fine sediments which also imply that the sediments are not too exposed for reworking or winnowing in this setting (Okoyen, 2006).

\section{Graphic Kurtosis}

The graphic kurtosis ranges from $0.14 \phi$ (very platykurtic) to $1.4 \phi$ (leptokurtic) with an average of $1.15 \phi$ (leptokurtic) which indicates that the sediments are dominantly leptokurtic in nature. This suggests that centre is better sorted than the end.

\section{Mode of transportation}

According to Visher (1969) mode of transportation of sediment can be determined from the graphical mean grain size using the following criteria;

Suspension-Finer than $3 \phi$

Saltation- $0.5-3 \phi$

Traction-coarse than $0.5 \phi$

The samples 1-10 from Famgbe River were transported by saltation and traction. This can be deduce from the grain size mean ranging from $-0.74 \phi$ to $0.77 \phi$ with an average of $0.17 \phi$ from the cumulative frequency curve.

\section{CONCLUSION}

The present work was carried out to study the nature of grain size distribution of sediments along the Famgbe axis of the River Nun. Various statistical parameters (i.e. mean size, sorting, skewness and kurtosis) are evaluated. The textural parameters indicates that the sediments were very coarse to coarse, poorly sorted to moderately well sorted, nearly symmetrical to dominantly positively skewed and very platykurtic to leptokurtic in nature.

The mean size indicates that the sediments were deposited in high energy conditions. The moderately well sorted character of sediments indicates the dominant moderately well sorted character infers that, the transportation system responsible for the deposition was very turbulent where there is a lot of winnowing. The graphic skewed suggest that the coarse admixture exceeds the fine sediments which also imply that the sediments are not too exposed for reworking or winnowing in this setting.

From the above findings the follow can be infer

- That the sediments were deposited in high energy condition

- That the sediments may have been deposited in a fluvial environment

- That the sediments may have been transported by saltation and traction

- That the sediments were derived from a single provenance.

\section{REFERENCE}

[1] Abam, T. S. K. (2016). Engineering Geology of the Niger Delta. Journal of Earth Sciences and Geotechnical Engineering, 6(3): 65-89pp

[2] Akpokodje, E. G. (1987). Engineering Geological Characteristics and Classification of the Major Superficial Soils of the Niger Delta. Engineering Geology, 23: 193-211pp 
[3] Akpokodje, E.G., Avwenagha, E.O. and Tse, A. (2014). Geotechnical Properties of Subsurface Soils in Warri, Western Niger Delta, Nigeria. Journal of Earth Sciences and Geotechnical Engineering, 4 (1) : 89-102pp

[4] Amajor and Ngerebera (1990). Significance of compositional and textural properties of sand from Western Andoni and Imo Eastern Niger Delta Nigeria. Journal of Mineral Geology of science, 26(2): 231-245pp

[5] Amajor, L.C. (1989). Geological Appraisal of Groundwater Exploitation in the Eastern Niger Delta, (ed. C. Ofoegbu) Groundwater and Mineral Research of Nigeria: Braunschweig/Weisbadan, Friedrvieweg and Sohn: 85-100pp [6] Asseez, O. L. (1989). Review of the Stratigraphy, Sedimentation and Structure of the Niger Delta. In: Kogbe, C. A. (Ed) Geology of Nigeria, Rock View (Nig.) Ltd.: 311-324pp

[7] Avbovbo, A. A. (1978). Tertiary Lithostratigraphy of Niger Delta. Bulletin of American Association of Petroleum Geologist, 62: 297-306pp

[8] Burke, K., Dessauvagie, T. F. J. and Whiteman, A. J. (1972). Opening of the Gulf of Guinea and Geological History of the Benue Depression and Niger Delta.

[9] Doust, H. (1989). The Niger Delta: Hydrocarbon Potential of a Major Tertiary Delta Province Proceeding. KNGMS Symposium” Coastal Lowland Geology and Geo-technology” Dordrecht: Kluwe, 203-212pp.

[10] Environs, Southeastern Nigeria: Evidence from Field study and Graunlometric Analysis. Journal of Natural Sciences Research, 5(17) : 44-53pp

[11] Etu-Efeotor (1998). Textural composition and provenance of traction sediments of the River Niger in Nigeria. Global Journal of pure and applied science, 5(3): 385-395pp

[12] Evamy, D.D., Haremboure, J., Kamerling, W.A., and Rowlands, P.H.(1978). Hydrocarbon Habitat of Tertiary Niger Delta. American Association of Petroleum Geologists Bulletin, 62: 1-39pp.

[13] Ezenwaka, K.C., Odoh, B.I and Ede, T. A. (2015). Lithofacies Analysis and Depositional Environments Of Eocene Nanka Sand as Exposed at Alor and

[14] Folk, R. L. (1966). A review of grain size parameters. Sedimentology, 6: 73-79p

[15] Folk, R. L. and Ward, W.C. (1957). Brazos river bar: a study in the significance of grain size parameters. Journal of Sedimentary Petrology, 27:3-26pp

[16] Iloeje, N. P. (1972). A New Geography of West Africa, Longman Group Limited, Nigeria, 19-20pp

[17] Inyang P. B. (1975). Climate in Ofomata Gek(Ed), Nigeria in maps Eastern States. Ethiope Publishing House Benin City, 25-26pp

[18] Krumbein, W. C. and Sloss, L. L. (1963). Stratigraphy and Sedimentation, Second Edition, Freeman and Company, San Francisco. 660pp

[19] Murat, R. C. (1972). Stratigraphy and paleogeography of the Cretaceous and lowerTertiary in Southern Nigeria. In Proc. of the Conf. on African Geology held at Ibadan, Nigeria, 251-266pp

[19] Nature, 233(38), 51-55.

[20] Ojo, O.J., Depositional Environment and Petrographic Characteristics of Bida Formation around Pategi, Northern Bida Basin, Nigeria, Journal of Geography and Geology, 224-241pp

[21] Ojo, S.O., Olege, K. O. and Ezechukwu, E. C. (1992). Countdown to Senior School Certificate Examination, Geography. Ibadan, Evans Brothers, Nigeria Limitd.

[22] Okoyen, B. Y. (2006) Comparative Textural and Compositional Analysis of Sediments in River Nun (Amassoma) Bayelsa State and Orashi (Mbiama) River State. Unpublished Bsc Thesis Niger Delta University Wilberforce Island, Bayelsa State, 44-47pp

[23] Parthasarathy, P., Ramesh, G., Ramasamy, S., Arumugam, T. (2016). Sediments Dynamics and Depositional Environment of Coleroon River Sediments, Tamil Nadu, Southeast Coast of India. Journal of Coastal Sciences, 3(2):1$7 p p$

[24] Reijers, T. J. A. (1996). Selected Chapters on Geology: with notes on Sedimentary Geology, Sequence Stratigraphy and three case studies and a field guide. S.P.D.C. Corporate Reprographic Services, Warri, Nigeria. 197pp

[25] Shettima, B., Tanko, I.Y., Kuku, A.Y. and Adams, F.D. (2016). Sedimentology and Plaeoenvironment of Deposition of the Deba-Fulani Members of Pindiga Formation in the Gongola Arm of the Upper Benue Trough, Northeastern Nigeria. Arid Zone Journal of Engineering Technology and Environments, 12: 17-30pp

[26] Short, K. C., and Stauble, A. J.,(1967). Outline of geology of Niger Delta. American Association of Petroleum Geologists Bulletin, 51: 761-779pp.

[27] Visher, G.S. (1969). Grain Size Distribution and Depositional Process. Journal of Sedimentary Petrology, 39(3): 1074-1106pp

[28] Weber, K.J. and Daukoru, E. (1975). Petroleum Geology of the Niger Delta. 9th World Petroleum Congress Proceeding, 2:209-221pp 